\title{
The influence of stimulus uncertainty in a reaction time situation
}

PHILIP TOLIN

UNIVERSITY OF IOWA

Forty-eight $S$ s participated in an experiment designed to test the effects of stimulus uncertainty on reaction time(RT). The results were interpreted as supporting the notion of increasing RT as a function of monitoring difficulty, rather than stimulus uncertainty.

A number of investigators have examined the effects of stimulus uncertainty-or difficulty in predicting the location or occurrence of signals-on reaction time (RT), and have generally found RT to be directly related to the number of stimulus alternatives. Garner (1962), however, has proposed that it is not the variation of stimulus uncertainty per se but, rather, a concomitant variation of some other factor such as the expected number of response discriminations that is the primary determinant of variations in $\mathrm{RT}$.

The present experiment represented an attempt to assess the relative importance of stimulus uncertainty under conditions in which signals might derive from (a) any of four equally probable alternatives (Group 4:4), (b) any of eight equally probable alternatives (Group 8:8), and (c) any of eight possible sources but were, in fact, chosen from only four (Group 4:8). Cronbach (1955) has noted that $\mathrm{S}$ often enters the experimental situation with his own, subjective, a priori expectancies, and that S's responses are primarily determined by these built-in expectations. As training proceeds, however, it can be expected that subjective stimulus uncertainty will begin to approximate the objectively determined measure.

Following this rationale, it was assumed that, since the amount of stimulus uncertainty at the beginning of the experimental procedure was the same for Groups $4: 8$ and $8: 8$ ( 3.0 bits), i.e., since the same number of stimuli were presented to both groups, the RTs for these groups would be similar and they would be slower than those of Group $4: 4$ (the stimulus uncertainty for this group being only 2.0 bits). If stimulus uncertainty were the crucial factor in determining the relative RTs in this study, however, it would be expected that, as Ss in Group 4:8 learned the actual probabilities of occurrence of the stimuli, the RTs of these Ss should diverge from those for Group 8:8 and approach those of Group 4:4.

\section{Method}

The Ss, introductory psychology students, were run in three independent groups of 16 Ss each (eight males, eight females). Group 4:4 was presented with four digits on each trial, while Groups $8: 8$ and $4: 8$ were presented with the same digits plus two at either side. The stimuli were presented in a 2-field tachistoscope such that
S's response would turn off the field and reactivate a white pre-exposure field. The intertrial interval was 6 sec. following the S's response. On every trial, one of the digits was underlined; the S's task was to call out this digit. Times from the onset of illumination of the stimulus field to S's vocalization of the underlined stimulus were recorded in milliseconds. Instructions to the Ss emphasized that responses were to be made as quickly as possible.

For Groups $4: 4$ and 8:8, each of the presented digits served as the signal equally often, while for Group $4: 8$ only the central four digits could be underlined. The signals for Group 8:8 were arranged such that each of the four central digits was underlined once in each block of eight trials. The other signals were randomized about these, with the restriction that they occurred equally often. Signals for the other groups were yoked to those of Group 8:8, so that, on the trials on which the latter group was presented any of the four central digits, the other groups were given the same signals. RTs on these trials only were analyzed.

The Ss were given two practice trials, followed by 88 test trials, although, as indicated above, data from only 44 were analyzed.

\section{Results}

Median RTs for each $S$ were obtained for the four critical trials within each of 11 blocks of eight trials. Arithmetic means of these medians are illustrated in Fig. 1. As shown in the figure, Groups 4:8 and 8:8 did not differ reliably $(F=0.04, d f=1 / 30)$. The performance of Group 4:4 was significantly more rapid than that of

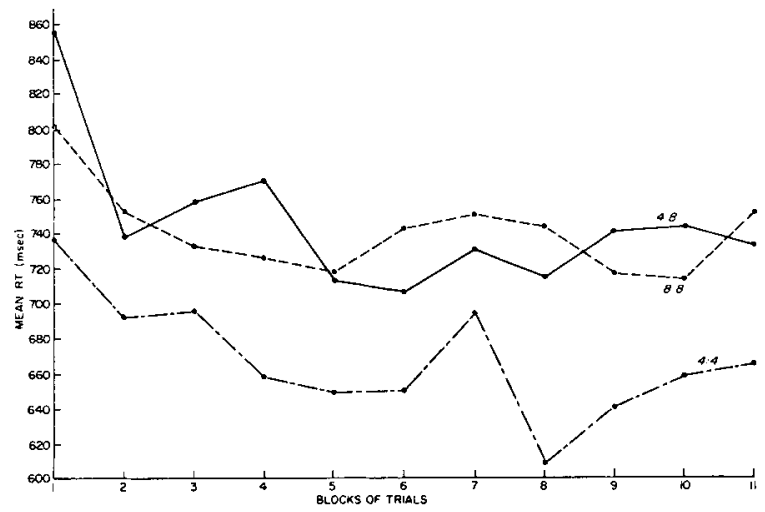

Fig. 1. Mean RTs, during 11 blocks of trials, of Groups 4:4, $4: 8$, and $8: 8$. 
Groups $4: 8$ and $8: 8$ combined $(F=12.96, d f=1 / 46$, $\mathrm{p}<.01)$. There were no significant interactions, either between Groups 4:8 and 8:8 ( $F=1.60, d f=10 / 300)$ or between the composite of these groups and Group 4:4 $(F=1.25, d f=10 / 460)$. Finally, the decrease in $R T$ as a function of stage of practice was significant $(F=10.75$, $\mathrm{df}=10 / 460, \mathrm{p}<.01)$.

\section{Discussion}

The data appeared to indicate that stimulus uncertainty was not a crucial factor in determining the relative RTs of the three groups in the present study. Had stimulus uncertainty been of importance, then an interaction would have been expected in which the RTs of Ss in Group 4:8 diverged from those of Ss in Group 8:8 and approached those of Ss in Group 4:4. As noted above, such an interaction was not obtained.

Verbal reports of the Ss at the end of testing tended to confirm this conclusion. The Ss in Group 4:4 indicated that by midway through the session their expectancies had begun to approximate, if not match, the actual probabilities of occurrence of the signals and that, by the end of the session, they had ceased to expectany of the zeroprobability stimuli. Yet the RTs of this group continued to be comparable to those of Group 8:8 and slower than those of Group 4:4.

It would appear, also, that the amount of response uncertainty did not determine RTs in this study. As the stimulus uncertainty in Group 4:8 decreased, a similar decrease should have occurred in the amount of response uncertainty, since knowledge that only the four central digits were to be underlined should have permitted Ss to attend more closely to these digits, thereby restricting the number of effective alternative responses.

The results of this study indicate that the RTs were perhaps largely determined by the size of the entire stimulus array, and that Ss were influenced by the presence of elements with zero probability of being underlined. It is this monitoring difficulty which may, in fact, be responsible for many of the results often attributed to stimulus uncertainty.

\section{References}

Cronbach, L. J. On the non-rational application of information measures in Psychology. In H. Quastler (Ed.), Information theory in psychology. Glencoe, Ill.: The Free Press, 1955.

Garner, W. R. Uncertainty and structure as psychological concepts. New York: Wiley, 1962. 